\title{
Formaldehyde and VOC Emissions from EFBMF Panel for Sound Absorption Material
}

\author{
Samsudin E. M. ${ }^{1 *}$, Ismail L. H. ${ }^{1}$, Nasidi I $\mathbf{N}^{1}$, Bakar H. A. ${ }^{1}$, Elgadi A. A. ${ }^{1}$ \\ ${ }^{1}$ Universiti Tun Hussein Onn Malaysia, Faculty of Civil and Environmental Engineering, Parit Raja, 86300 Batu \\ Pahat, Johor, Malaysia \\ * E-mail:emedya.samsudin@gmail.com
}

\begin{abstract}
Formaldehyde and volatile organic pollutants are among two major indoor air pollutants that threatens the use of wood-based building mate-rials acceptance. Issue relating to indoor air pollution could not be ignored when dealing with forestry-by product materials since the source of pollutant is exists from the wood binder and these issues are very important from the indoor environmental quality point of view. In this research, Empty Fruit Bunch Mesocarp Fiber (EFBMF) acoustic panel was produced as a sound absorption material for indoor acoustic application. Monitoring of pollutants emitted from EFBMF panels were done in two different stages; during fabrication and during applica-tion in the acoustic chamber under room conditions. In the first stage, chemical compositions of the testing materials were measured in three (3) different conditions-raw fiber, sodium treated fiber and panel fiber using X-ray fluorescence (XRF) evidenced low chemical compositions contained by both EFB and mesocarp fibers. Measurement on gas emissions during fabrication using IEQ equipment evidance the presence of formaldehyde and VOC emissions in the ambient air during hot press work. Meanwhile in the acoustic chember, formaldehyde concentration were found increased with time while none VOC was detected to released to the surrounding environment during the 24 hours of measurement. Analysis shows that the treat from formaldehyde emission is lower for EFBMF panel during application compared to during fabrication.
\end{abstract}

Key words : EFB, formaldehyde, MF, volatile organic compound.

\section{INTRODUCTION}

Nowadays, several toxic gasses, hazardous substances and pollutants from biological origins are found to contaminate indoor environment [1]. Indoor air pollution occurs in many ways such as from normal biological process by human and animals, from combustion appliances used inside building, the use of consumer products from building materials, furnishing and insulations, cigarette smoke, soil underneath and around building as well as from other appliances installed to the building to support the building operation [2]. According to Saad et al. [3] among the major types of pollutants that are available indoor are ozone $\left(\mathrm{O}_{3}\right)$, volatile organic compound (VOC), carbon monoxide (CO), nitrogen dioxide $\left(\mathrm{NO}_{2}\right)$, carbon dioxide $\left(\mathrm{CO}_{2}\right)$, particulate matter $\left(\mathrm{PM}_{10}\right)$, oxygen $\left(\mathrm{O}_{2}\right)$, temperature and humidity. Among those sources, building materials are one of the source to indoor air-borne pollutant and has been defined as among the major contributors to formaldehyde emissions and volatile organic compounds (VOC), which have significant effects to human health [4]; [5].

Factors that contribute to the adverse health effect from building materials emissions are the concentration levels of the pollutants, exposure time and the existing health condition of the occupant during exposure time and thereafter. Even though low concentrations are unable to show immediate health effect to human, continuous exposure may cause serious health problems especially to children [2]. Building materials which consist of wood-base materials normally used urea formaldehyde as a binder in the production such as fibreboard, particle borad, hardwood plywood and medium density fibreboard. The use of this amino-resin binder has led to several health issues that has been an obstacle to their acceptance by the public [6], [7], [8].

Monitoring of the environmental impact from building materials is very important because the building industry is one of the largest industry sector [9] in most of the developed and developing countries. Emissions of harmful gasses from materials installed inside a building are very crucial since most people spend more than $80 \%$ of their time indoors. This has higher risks and implications than from outdoor pollutions [2]. According to Saad et al. [3], as the concentrations are five times higher in the normal ambient air due to gases or other substances, the indoor air is considered as polluted. When the amount of these gases exceeds permissible limits, their exposure can be harmful and hazardous to human. Irritation of the eyes, nose and throats, headaches, dizziness, fatigue, asthma and humidifier fever are some of the most common immediate health problems resulting from exposure to indoor air pollutants [3]. Formaldehyde and VOCs are among the major indoor air pollutants in building [10], [4]. The United States Environmental Protection Agency (EPA) is putting emphasis on understanding and controlling common pollutants inside buildings to reduce the risk of indoor health 
concerns. Therefore, knowledge on the emission characteristics is very important to monitor the level of pollutant concentrations of the indoor air and the associated health risk for effective source control and maintaining a sustainable building environment [5].

Indoor air pollutants that are hazardous to human are formaldehyde and other VOC pollutants such as benzene, polycyclic aromatic hydrocarbons $(\mathrm{PAH})$, trichloroethylene and tetrachloroethylene. Even though most of these pollutants are emitted continuously from building materials, the emissions rate and concentration levels decrease over time [2]. Formaldehyde particularly can lead to sensory irritations such as to eyes, nose and throat. The average exposure shall not exceed $0.1 \mathrm{mg} / \mathrm{m}^{3}$ within an average of 30 minutes. Affect from exposure to excessive Benzene and Polycyclic aromatic hydrocarbon can lead to leukemia and lung cancer while exposure to trichloroethylene and tetrachloroethylene can damage our internal organs such as kidney and liver. Considering the adverse effects to health, it is very important to define the source of pollutants from the building materials when the risk of occurrences from the potential pollutants might exist.

\subsection{Volatile organic compound (VOC)}

Volatile organic pollutants are any chemical components that contain at least one carbon atom and hydrogen in their molecular structure [2]. Volatile organic pollutants are categorized into different classifications; very volatile organic compounds (VVOC), volatile organic compound (VOC), semi volatile organic compound (SVOC) and nonvolatile organic compound (NVOC) [2]; [11] and [1]. These organic compounds have different boiling points with VVOCs boiling points ranging from $<0{ }^{\circ} \mathrm{C}$ to $50-100{ }^{\circ} \mathrm{C}$, VOCs boiling points ranging from $50-100{ }^{\circ} \mathrm{C}$ to $240-260{ }^{\circ} \mathrm{C}$, while SVOCs boiling points ranging from $240-260{ }^{\circ} \mathrm{C}$ to $380-$ $400{ }^{\circ} \mathrm{C}$. The wide variety of components in this pollutant has made it one of the most dominant indoor airborne pollutants in building [1].

Exposure to volatile organic pollutants can lead to several health effects such as eye irritation, headache and nausea [3]. Moreover, high concentration and continuous exposure of individual VOCs leads to indoor air quality (IAQ) problems [12] with high toxic content in this pollutant may also cause other respiratory diseases which could lead to the risk of cancer to human [13]. There are hundreds of volatile organic pollutants exist in our indoor air atmosphere [12].

The United States Environmental Protection Agency listed out major sources of VOC in building, commonly originating from household products, building materials and furnishing, office equipment and in graphics and craft materials such as glue and adhesive [13]. Some of the most common VOCs are methane, ethane, tetrachloroethane, methyl chloride, and various chlorohydrocarbons and perfluorocarbons [14]. High volatility made VVOC emission rates is higher than VOC and SVOC. However VVOC and VOC emission rate usually will decrease substantially in the first few weeks or months while
SVOC will continue to be emitted throughout the product life cycle [11].

Even though most of the wood-based building materials release higher VVOC levels, particularly formaldehyde, to the air resulting from the chemical reaction between the resin with moisture [15], but other detectable VOCs were also determined [16] especially during the production of the wood-based material [17]. Research by Kim et al. [18] found that the use of coating material on medium density fiberboard (MDF) causes higher total volatile organic compound (TVOC) emission factor.

Research by Jun \& Shuang, 2012 [19] measured the VOC emission from wood-based panel namely the high density fiber board (HDF), medium density fiberboard (MDF) and PVC veneer medium density fiberboard (MDF veneer) in an environmental chamber. This research found that toluene, ethylbenzene, styrene and $\mathrm{m}$, p-xylene is the main VOC emitted from those three tested fiberboards specimens, which is categorized as aromatics VOC. Moreover, findings also show that after 28 days, their concentration levels decreased significantly, especially toluene and for the other compounds, their emissions stabilised. The emission rates of aromatic compounds of VOCs also increase significantly with the increase in temperature and pressing time [20]. This shows that different manufacturing stage of wood-based products also contributes to certain emission level of this pollutant. Research by He, Zhang \& Wei [21] found 34 individuals VOCs during the manufacturing stage of wood-based panels. This shows that the emission on VOC started as early as during the wood-based panel production and will continue throughout their life cycle.

\subsection{Formaldehyde}

Formaldehyde has been used widely in the manufacturing of building materials and various household by-products [13]. Formaldehyde is also known as a major VOC pollutant and is categorized as very volatile organic compounds (VVOC) [11]. This pollutant can be found in most building materials and furnishing such as particleboard, medium-density fiberboard, plywood, carpet, furniture, tobacco smoke and in some of combustion appliances [2].

In most of forestry-based product of building material and furnishing, formaldehyde has been widely used as an essential component of resin binder [22] and [2]). As a major wood binder, formaldehyde has excellent bonding properties and is inexpensive, and therefore widely used in industry. The presence of formaldehyde in the UF-bonded materials causes the gas to be trapped in the structure of the substrate or dissolved in the moisture content of the board. This will lead to the formaldehyde emissions into the air [23].

According to Salem et al. [8], formaldehyde emissions in buildings are mainly from wood-based panels and flooring materials installed inside the building. Formaldehyde, $\mathrm{HCOH}$, is a colorless and a strong smelling gas, which is flammable at room temperature. Agency for Toxic Substances and Disease Registry (ATSDR) stated that level of formaldehyde 
concentration is normally higher indoor (ranges between 0.02 $\mathrm{ppm}$ to $4.0 \mathrm{ppm}$ ) compared to outdoors (in between 0.0002 $\mathrm{ppm}$ to $0.2 \mathrm{ppm}$ ). This agency has stated that exposure of 0.1 $\mathrm{ppm}$ to $0.5 \mathrm{ppm}$ to human can lead to nasal and eye irritation, neurological effects and increases the risk of asthma and/or allergy. Exposure of $0.6 \mathrm{ppm}$ to $1.9 \mathrm{ppm}$, formaldehyde can cause eczema and changes in lung functions. This agency also reported that based on study made by the Department of Health and Human Services (HHS) in 2011, formaldehyde has been describes as a known human carcinogen, which can lead to the risk of cancer [24].

Numerous studies have been conducted to evaluate formaldehyde emission from various wood-based materials [25], [8] and [6]. Research by Salem et al. [8], evaluated formaldehyde emission (FE) and content (FC) from particleboard $(\mathrm{PB})$, medium and high density fiberboard (MDF and HDF), plywood (PLW) and several other flooring materials such as HDF laminate, solid wood, solid bamboo and polyvinyl chloride (PVC) using different test methods. This research has found that the emissions level of most products is below the permissible limits recognized by their local authorities. The thickness and types of panels also influenced the emission levels. Thicker samples tend to emits higher concentrations, with PVC having the highest amount of FE [8].

Böhm et al. [25] investigated formaldehyde emissions from manufactured flooring and blockboard made from different wood species. This research also investigated the effects of different wood-adhesive (urea-formadehyde and phenol-formaldehyde) used in the manufacturing of wood-based bulding products. It was reported that the formaldehyde emission values are significantly affected by the wood species, plywood type and their thickness. Solid woods measured between $0.0068 \mathrm{ppm}$ to $0.0036 \mathrm{ppm}$ and $0.084 \mathrm{mg} / \mathrm{m}^{2} \mathrm{~h}$ to $0.014 \mathrm{mg} / \mathrm{m}^{2} \mathrm{~h}$ of formaldehyde. Measurements of formaldehyde emission for engineered flooring with polyvinyl acetate showed an initial value from $0.006 \mathrm{mg} / \mathrm{m}^{3}$ while for painted birch blockboard the value ranged from $0.048 \mathrm{mg} / \mathrm{m}^{3}$. This research also found that painted blockboards emits higher formaldehyde levels compared to uncoated boards. And formaldehyde emission for all materials was found to decrease after 2 weeks of measurements [25].

In Kim \& Kim [6], different resins were used to produce medium density fibreboard (MDF) for use in laboratory testing. This research used urea formaldehyde (UF) resin, melamine-formaldehyde (MF) resin and the co-polycondensation resin of urea-melamine-formaldehyde (UMF) and melamine-formaldehyde with a molar ratios of $1.25(\mathrm{~F} / \mathrm{U})$ for UF resin, $1.75(\mathrm{~F} / \mathrm{M})$ for $\mathrm{MF}$ resin and 0.65 (F/M and U) for UMF respectively. Additionally, $25 \%$ of ammonium chloride (hardener) and $44 \%$ of wax solution was used for waterproofing, being added into the mixture to accelerate the curing process and to control the moisture content of the end product. It was found that formaldehyde emission levels for MDF made with UF resin obtained the highest concentration compared to MDF made with MF resin.
This research also showed that when the MF content increased, the FE decrease significantly. However, the used of MF resin as adhesive for wood-based panels is costly and could cause the production cost to increase significantly compared to the use of UF resin which is cheaper and more economical [6].

Study by Parthasarathy et al. [26] defines the relationship between temperature and humidity on formaldehyde emissions levels in temporary housing units. The temporary housing units (THU) are the temporary houses supplied by the United State Federal Emergency Management Administration (FEMA) to the hurricane victims in the United States. The sources of formaldehyde were identified as being contributed by the building materials and furnishing inside the THU. From several laboratory experiments conducted on test specimens obtained from the THU, it was found that increases in temperature caused the formaldehyde emission levels to increase. Likewise, high humidity factor also contributed to higher formaldehyde emission of the test materials. This research also obtained a linear regression model between inverse temperature coefficients with $\log$ relative humidity coefficients. All tested samples from the THU obtained R2 values above $97 \%$ confidence level [26].

The risk on formaldehyde emissions is very common from wood-based building materials and products. Formaldehyde not only emits from the end-product but could exist as early as during production. It is vital to monitor their emission in both stages (production and application) when dealing with wood-based building material. Therefore, the main purpose of this study is to evaluate the environmental effects from the new EFBMF acoustic panel in term of formaldehyde and VOC emissions. Therefore, monitoring on the presence of both pollutants in the ambient air were done to EFBMF panels during fabrication stage in the laboratory and during application in the acoustic chamber.

\section{METHODOLOGY}

In this study, the determination of environmental effects of EFBMF acoustic panel was done based on two parts. Part 1 was determination of chemical composition of the fibers and EFBMF acoustic panel in three different conditions (raw fibers, composition of fibers after treatment and composition from the EFBMF panel). Meanwhile in the Part 2, monitoring and measurement of formaldehyde and VOC emission were done in two different stages; during hot pressing of EFBMF acoustic panel sized $30 \mathrm{~cm} \times 27 \mathrm{~cm}( \pm 0.9 \mathrm{ft}$ square $)$ and during application of the panels (sized $\pm 10.1 \mathrm{ft}$ square) in room conditions.

\subsection{Chemical Properties Measurement}

$\mathrm{X}$-ray fluorescence (XRF) testing was done to investigate the chemical composition of the testing materials. Measurement of chemical composition of both EFB and MF samples were done in three (3) different conditions-raw fiber, sodium treated fiber and panel fiber. This testing is done using standard less and quantification program in the Advance Environmental Analysis Laboratory, FKAAS, UTHM. 
Pressed pallets were prepared before performing the X-ray screening on the samples. Samples were first grounded and sieved, passing $68 \mu \mathrm{m}$-sieve sizes. Each pallet requires at least $8 \mathrm{~g}$ of sample dust and $2 \mathrm{~g}$ of wax (boric acid) to form the pallet. Both weighed sample dust and wax are mixed in the same beaker thoroughly. Before pouring the mix into the die set, all die set components that attached to the pallet must be cleaned and polished using alcohol. This is to ensure that the pressed pallet does not contain contaminants.

Figure 1 shows the press pallets prepared in this research. $\mathrm{XRF}$ testing in the laboratory was conducted by the authorised technical staff in the laboratory using Bruker Advance X-Ray Solutions equipment and results were obtained from SPECTRA Plus programs attached to the equipment.

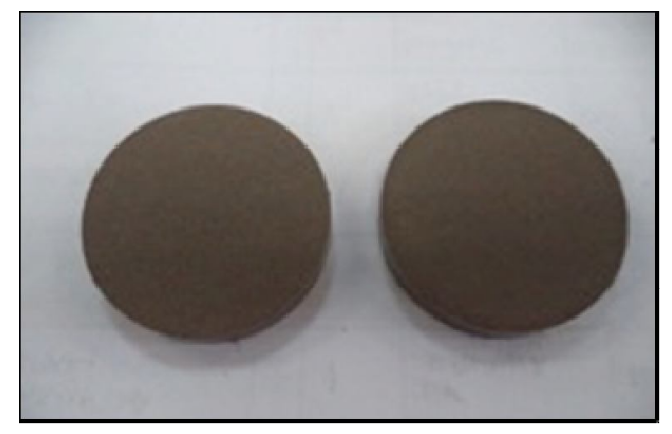

Figure 1: Pressed pallet

\subsection{Gas Emission Measurement}

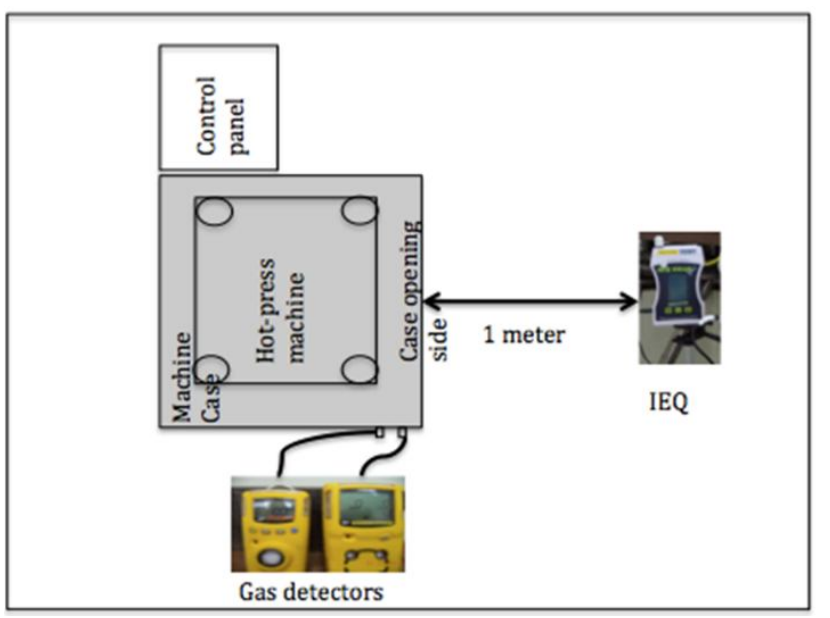

Figure 2: Equipment setting for gas emission and indoor air quality measurement during hot pressing

Measurement for gas emission is done in two different stages, during fabrication (hot pressing work) and during application in the acoustic chamber under room condition. In the first measurement, gas detectors were attached to the special casing installed to the hot press equipment to trap the emitted gasses. All bottom opening of the case were sealed with aluminium foil to ensure that gasses will exit only from the front of the casing where the indoor environmental quality (IEQ) equipment is installed. During measurement, IEQ equipment was installed in distance of 1 meter from the hot press machine case opening to measure the air quality during the hot pressing procedure (refer to Figure 2).

In this investigation, two types of gas detectors were used- the single detector with Sulfur Dioxide (SO2) sensor and multiple detectors with hydrogen sulfide (H2S,), carbon monoxide (CO) and lower explosive limit (LEL) sensors. Meanwhile the IEQ equipment was equipped with seven sensors namely temperature, relative humidity $(\mathrm{RH})$, formaldehyde $(\mathrm{CH} 2 \mathrm{O})$, oxygen $(\mathrm{O} 2)$, sulfur dioxide ( $\mathrm{SO} 2)$, carbon dioxide $(\mathrm{CO} 2)$ and volatile organic compound (VOC). These parameters are among the main parameters required for indoor quality assessment by the Department of Safety and Health (DOSH). However, this research will focus on formaldehyde and VOC emission during both stages.

Measurements were done in real time before and during the hot pressing procedure. However, before running the measurements, setup and live readings from both equipments were crosschecked for the same sensor. This is to ensure that both devices are working correctly and ready for use. To ensure the reliability of data, this procedure was undertaken only when there are no other activities held at the same time in the laboratory. The used of personal protection equipment (PPE) such as gas mask and gloves are strictly adhered to during the hot pressing procedure and gas measurement. This is to prevent from possible health risk due to unknown gas emissions from the heating process. Real time measurements were set to the IEQ equipment and the data logger recorded data of all parameters displayed by the equipment. Time interval for data logger is set for 30 seconds throughout the measurement period. Meanwhile, the gas detectors gave an audible "beep" when gases are detected. Figure 3 shows the measurement setup in the acoustic chamber during application stage.

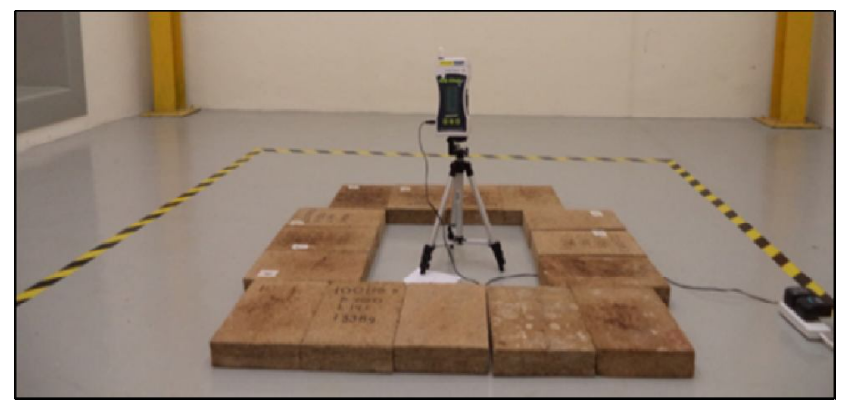

Figure 3: Measurement of gas emission in the acoustic chamber

\section{RESULTS AND DISCUSSION}

\subsection{Analysis on chemical compositions of fibers}

Determination on chemical compositions of fibers was done at different stages; raw fibers, fibers after being treated with sodium hydroxide $(\mathrm{NaOH})$ and compositions from the fabricated panels. This was purposely done to know the chemical concentration inside each of the fibers and at different processing stages by XRF analysis using pressed pallets technique in the Environmental Analysis Laboratory, Faculty of Civil and Environmental Engineering, UTHM. 
Among the major chemical elements contained in the raw EFB are potassium, ferric (iron), calcium, silicon (silica) and chlorine. Untreated EFB also contains other chemical elements such as sulfur, phosphorus, aluminum, magnesium, carbon and zinc, but the amount of concentrations is less than 1\%. Research by Abdullah, Sulaiman, and Gerhauser [27] using Electron Dispersive X-ray Spectroscopy (EDX) technique on EFB also found almost similar chemical elements in this fibre. After EFB was treated with $2 \%$ of sodium solutions $(\mathrm{NaOH})$, chemical reaction between the solutions and the raw fiber had slight changes on the chemicals concentration contained by the fiber.

This research has found that the percentage of major chemical elements such as silica, calcium and iron increases but the percentage of potassium and chlorine decreased. Since lignin is the outer layer of EFB, therefore the used of sodium treatment has caused the lignin content of this fiber to decreased which is the reason for the loss of potassium and chlorine from the fiber. The same result was also obtained by Ariffin et al. [28] where chemical treatment using $0.1 \mathrm{M}$ of $\mathrm{NaOH}$ and $0.5 \mathrm{M}$ of $\mathrm{NaOH}$ caused the lignin content to reduce from $15.17 \%$ for the raw EFB to $14.21 \%$ and $13.20 \%$ respectively. After EFB panel was fabricated, the amount of chlorine concentration was found to increase. This was due to the use of chemical hardener $\left(\mathrm{NH}_{4} \mathrm{CL}\right)$ being added in the wet fiber mixture to accelerate the hardening process of the acoustic panel after hot pressing.

The same chemical analysis was done to MF at all processing stages in the laboratory. However, loose powder technique was used to the raw MF since few attempts using pressed pallet technique for the same XRF analysis of this fiber failed to produce any result. Even though loose powder technique successfully produce the expected result, the composition of chemical elements was found to be slightly lower compared to the pressed-pallet technique. Besides, this technique was also unable to detect the composition of carbon in the fibre. Therefore, EDX analysis was done to the same sample as the supporting data.

MF contained high silicon dioxide in the fiber element compared to the other chemical elements due to more silica bodies were found attached to the MF surface especially on the raw fiber. Other than that, MF also contains potassium, calcium, ferric and chlorine as their major elements. Furthermore, EDX analysis on the same sample type shows that raw MF constitutes of $67.44 \%$ of carbon, $26.67 \%$ of oxygen, $2.92 \%$ of potassium, $1.09 \%$ of calcium, $0.81 \%$ of silicon $0.62 \%$ of magnesium and $0.46 \%$ of chlorine on the fiber surface. Different results were obtained since EDX only analyze the surface elements instead of the whole fiber elements. However, in the loose powder technique, the presence of $98 \%$ of $\mathrm{CH}_{2}$ was detected. High $\mathrm{CH}_{2}$ indicates the presence of lignin, which is similar to hardwood lignin [29]. This strongly suggests that this fiber contains high organic components that build up the fiber structure.

Furthermore, pressed-pallet technique was used to define the chemical components of treated MF. Results obtained using this technique to the treated fiber detected high silicon dioxide, calcium oxide, ferric oxide and potassium at $5.41 \%, 4.07 \%$, $3.94 \%$ and $1.36 \%$ respectively as their major components. Moreover, extracted fiber from the MF panel detected big increase in chlorine content which is due to the use of chemical hardener in the mixture. The amount of sodium and molybdenum content were also found to increase slightly compared to the treated fiber.

Based on the XRF analysis, accumulated chemical composition amount in both fibers at different processing stages is very low concentration. Both fibers can be said containing less than $20 \%$ of chemical compositions and showing that low risk associated with the hazardous chemical gas emissions to exists from this material. Research by Nordin et al. [30], Sabil, et al. [31] and in Ramli et al. [32] showed that EFB and MF consist of three major organic elements; hemicellulose, cellulose and lignin. The low chemical content determined by this research and high organic content evidence by earlier researchers provides a strong indication that the use of EFB and MF as building acoustic material might contributes to organic-based emission.

Table 1: Chemical compositions of fibers in different processing stages

\begin{tabular}{|c|c|c|}
\hline \multirow{2}{*}{ Fiber } & Category & $\begin{array}{c}\text { Chemical Compositions } \\
(\%)\end{array}$ \\
\hline \multirow{3}{*}{ EFB } & Raw & 17.24 \\
\cline { 2 - 3 } & Treated & 18.95 \\
\cline { 2 - 3 } & Panel & 12.40 \\
\hline \multirow{3}{*}{ MF } & Raw & 1.60 \\
\cline { 2 - 3 } & Treated & 18.09 \\
\cline { 2 - 3 } & Panel & 12.53 \\
\hline
\end{tabular}

\subsection{Formaldehyde emissions}

During measurement of formaldehyde emission during fabrication, the measuring device is placed at a distance of $1 \mathrm{~m}$ from the opening of the compressor cover. Throughout the measurement period, all other openings such as doors and windows were completely closed including the exhaust fans. Ventilation from the building's air conditioning system is left operated since this system is a centralise unit and not able to turned-off manually. This system was left to act as the indoor ventilation inside the laboratory in normal room condition. Figure 4 illustrates the average formaldehyde emissions level in the laboratory during the hot pressing work on from three (3) sets of measurement.

As can be seen from above figures, the first 150 to 200 intervals (within 75 minutes to 90 minutes) was the condition inside the laboratory before the fabrication work started. In normal conditions, the level of formaldehyde concentrations inside the laboratory is below $0.10 \mathrm{ppm}$. As the fabrication work started (whereby all machines are turned on and the UF spraying work on dry fibers commenced), it was observed that the formaldehyde concentration started to increase to more than $0.20 \mathrm{ppm}$. Later, when samples were uploaded into the hot pressed machine and the heat-pressing work began, the formaldehyde emissions level increased substantially to more than $1.0 \mathrm{ppm}$. 
The high heat applied to the wet fiber mixture causes the formaldehyde liquid to evaporate into the air. However, when the compression pressure of the hot plates was released and the heating temperature was gradually decreased, formaldehyde concentrations in the air decreased and reverted to normal levels. This cycle was repeated throughout the day during fabrication, as can be see in the graph. The peaks of the graph as can be seen in above figures represented the maximum emissions from the EFBMF acoustic panels when hot-pressed.

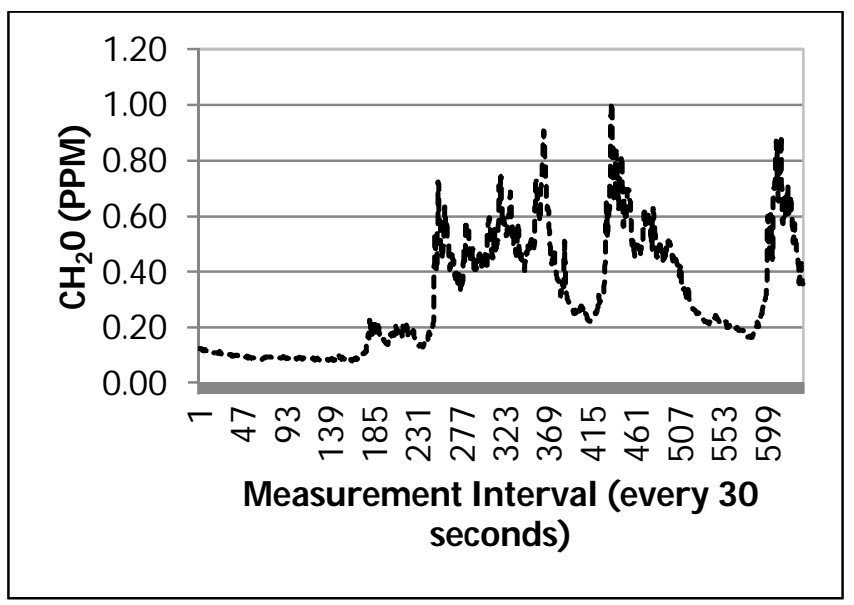

Figure 4: Average formaldehyde emission during hot press

Figure 5 showed the average formaldehyde emission level inside the acoustic chamber during the application stage. Measurement in the acoustic chamber was done for 24-hours in an empty room condition. During measurement doors and ventilation from the air conditioning systems were completely close to ensure that there are no other factors that might influence the measurements. Measurement was started by measuring the existing condition inside the empty chamber for 24-hours and followed by three (3) sets of measurement for occupied chamber (chamber containing $1 \mathrm{~m}^{2}$ of EFBMF acoustic panel laid on the floor).

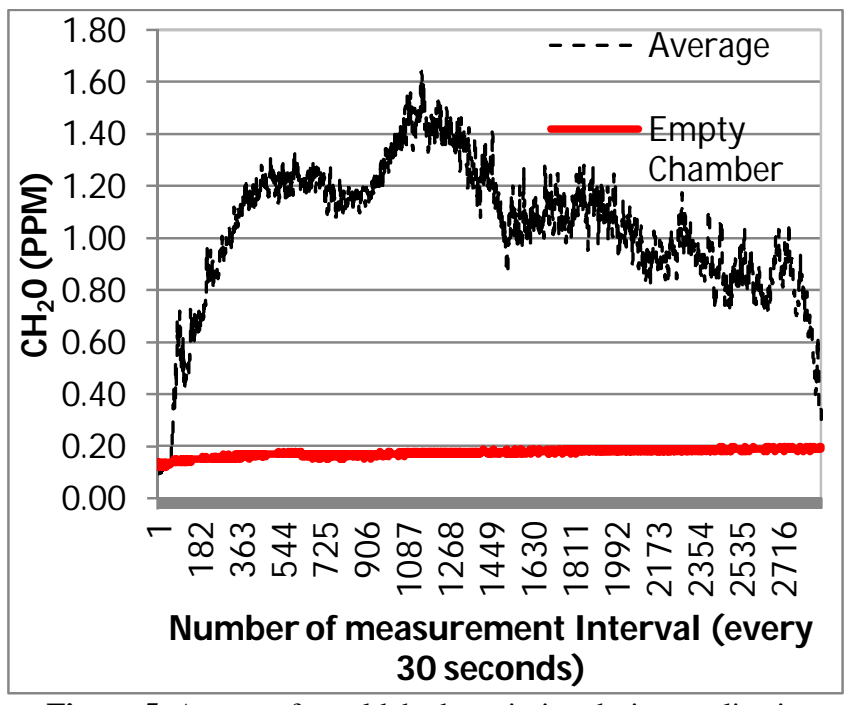

Figure 5: Average formaldehyde emission during application.
Measurement for empty chamber shows that in normal conditions, the formaldehyde concentration inside the chamber was less than $0.20 \mathrm{ppm}$. Existing formaldehyde concentration level in this room was slightly higher compared to previous stage because of several factors such as the smaller room volume and deactivated ventilation system inside the acoustic chamber. Moreover, small increase was observed in the formaldehyde concentration inside this chamber throughout the day Analysis showed that formaldehyde levels inside the acoustic chamber increased with time. The peaks emissions occur within 1200 and 2050 of intervals (approximately between $7.00 \mathrm{pm}$ to $3.00 \mathrm{am}$ ). Laterly, the concentration level decreased. The used of $1 \mathrm{~m}^{2}$ of EFBMF acoustic panel in the acoustic chamber caused the formaldehyde level to increase to more than $130 \%$ inside the chamber compared to existing formaldehyde level in measured when the chamber is empty. The high concentrations caused some discomfort experienced especially to the eyes when the chamber was opened to access. One of the factors that contributed to the high concentration level is the absence of space ventilation either natural or mechanical inside the chamber. This has led to the accumulation of formaldehyde emission in the air inside the chamber during measurement.

Research by Parthasarathy et al. [26] showed that formaldehyde concentration levels increase with increases in temperature and humidity. Since no mechanical ventilation had been activated in the chamber during measurement, the increase in temperature/higher temperature inside the acoustic chamber during measurement might be one of the reasons for higher formaldehyde emissions.

Monitoring process on the formaldehyde emissions level during fabrication shows that the average emission level is somehow very low and shows fewer hazards to occur during fabrication, which is at $0.31 \mathrm{ppm}$. Meanwhile, higher emissions were observed inside the acoustic chamber with an average value of $1.24 \mathrm{ppm}$, which is found to be above than 8-hours permissible limit suggested by DOSH at $0.75 \mathrm{ppm}$. Among the reasons for high emissions inside the acoustic chamber is due to the non-availability of the mechanical and natural ventilation as well as the disable of air conditioning system inside the chamber. Besides, smaller chamber volume compared to the fabrication laboratory used for measurement during application also plays an important role that influencing this result. Nonetheless, the size of the EFBMF acoustic panel used in the measurement also needs to be considered in the analysis.

Further analysis prior to the emission is done to define the emission factor per surface area of panel in both stages. Analysis in Table 2 confirms the emission level per surface area is higher during fabrication compared to during application. This evidence that high temperature used for heating the panel has caused more formaldehyde to evaporate and releases in the ambient air. The affects were also shown with high degree of discomfort experienced to eyes and nose during the fabrication work compared to during application. 
Thus, stringent safety precaution is required during fabrication when working with this chemical binder.

Table 2: Formaldehyde emission factor during fabrication and application stage.

\begin{tabular}{|c|c|c|c|}
\hline Stage & $\begin{array}{c}\text { Panel } \\
\text { size } \\
\left(\mathrm{ft}^{2}\right)\end{array}$ & $\begin{array}{c}\text { Average } \\
\text { emissions } \\
(\mathrm{ppm})\end{array}$ & $\begin{array}{c}\text { Emission factor } \\
\left(\mathrm{ppm} / \mathrm{ft}^{2}\right)\end{array}$ \\
\hline Fabrication & 0.9 & 0.31 & 0.344 \\
\hline Application & 10.1 & 1.24 & 0.123 \\
\hline
\end{tabular}

\subsection{Volatile organic compound (VOC) emissions}

Results for VOC emissions level during fabrication and application of EFBMF acoustic panels is shown in Figure 6. Measurements in the laboratory during fabrication work evidence the presence of VOC in all sets of measurements. The VOC concentrations were found increased tremendously when the sample was attached with high temperature during pressing. The increase in VOC concentration during hot presswork is suspected to come from the aromatic VOC being released from the fibers it selves at mentioned by Liu, Sheng and Zhu [20]. Meanwhile, measurement of VOC emission during application in the acoustic chamber founds that in a normal room temperature, none VOC was recorded emits into the ambient air during the 24-hours of measurement.

Since there are no reading were recorded in the acoustic chamber, its show that EFBMF acoustic panel used in this research released almost none VOCs during the 24-hours of monitoring during application. One of the reason is because those panels were not been coated with any finishing treatment or an over layer coating as is usually done to most readily available wood-based panel products in the industry.

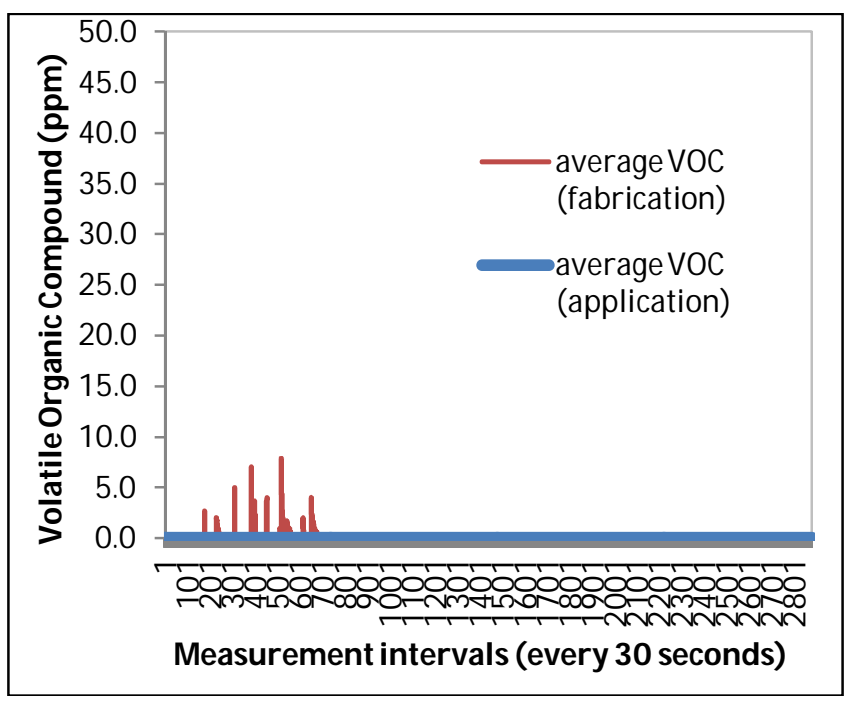

Figure 6: Average VOC emission during fabrication and application stage.

\section{CONCLUSION}

Sodium treatment and the use of $15 \%$ by weigh of urea formaldehyde and $1 \%$ of hardener into the fibers mixture had caused a slight change to the major chemical content of the fiber but does not change its major compositions. Earlier, investigation on the chemical compositions of EFB and MF has found that both fibers contained almost the same major elements such as potassium $(\mathrm{K})$, ferric $(\mathrm{Fe})$, calcium $(\mathrm{Ca})$, silicon $(\mathrm{Si})$, chlorine $(\mathrm{Cl})$, sulfur $(\mathrm{S})$, phosphorus $(\mathrm{P})$, aluminum (Al), magnesium (Mg), carbon (C) and Zink ( $\mathrm{Zn})$. Both fibers were found contained less than $20 \%$ of chemical compositions and consisted of organic components that are made of lignin, cellulose and hemicellulose layer, which is very similar to hardwood fiber components. The major concern of this research is to acknowledge the presence of formaldehyde and VOC emissions from the EFBMF acoustic panel. On site measurements to the formaldehyde emission levels from EFBMF acoustic panels showed that higher emission rate per surface area was obtained during fabrication compared to during application in a closed chamber. This is due to the effects of high temperature attached to the wet panel mixture causing the formaldehyde content in the resin binder to evaporate and be released to the ambient air. This finding showed that the risk on formaldehyde emission is three (3) time lower during application compared to during fabrication. The ambient temperature and relative humidity were also found to give a slight effect to the emission level of VVOC to the indoor environment. The presence of VOC is very low and almost nil in both stages. However, when the fiber was attached with high temperature, it caused the aromatic VOC to be released from the fiber into the surrounding.

\section{Acknowledgement}

Authors would like to thank the Ministry of Higher Education (MOHE) and Universiti Tun Hussein Onn Malaysia for sponsering this study and to Fundamental Research Grant Scheme (FRGS), vote 1524 for funding for this publication.

\section{REFERENCES}

[1] Godish, T. (2001). Indoor Environmental Quality. United States of America: Lewis Publishers.

[2] Hines L. A., Ghosh T. K., Loyalka S. K. \& Warder, R. C. Jr. (1993). Indoor Air Quality and Control. New Jersey: PTR Praentic Hall.

[3] Saad, S. M., Andrew, A. M., Shakaff, A. Y., Azuwan, M., Dzahir, M., Hussein, M., \& Ahmad, Z. A. (2017). Pollutant Recognition Based on Supervised Machine Learning for Indoor Air Quality Monitoring Systems. Applied Sciences, 7(8), 1-21 https://doi.org/10.3390/app7080823

[4] Niu, J. (2004). Some significant environmental issues in high-rise residential building design in urban areas. Energy and Buildings, 36(12), 1259-1263. https://doi.org/10.1016/j.enbuild.2003.07.005 
[5] Xiong, J., Zhang, P., Huang, S., \& Zhang, Y. (2016). Comprehensive Influence of Environmental Factors on the Emission Rate of Formaldehyde and VOCs in Building Materials : Correlation Development and Exposure Assessment. Environmental Research, 151, 734-741.

[6] Kim, S., \& Kim, H. J. (2005). Comparison of Standard Methods and Gas Chromatography Method in Determination of Formaldehyde Emission from MDF Bonded with Formaldehyde-based Resins. Bioresource Technology, 96(13), 1457-1464.

[7] Que, Z., Furuno, T., Katoh, S., \& Nishino, Y. (2007). Evaluation of Three Test Methods in Determination of Formaldehyde Emission from Particleboard Bonded with Different Mole Ratio in the Urea-formaldehyde Resin. Building and Environment, 42(3), 1242-1249.

[8] Salem, M. Z. M., Böhm, M., Srba, J., \& Beránková, J. (2012). Evaluation of Femission from Different Types of Wood-based Panels and Flooring Materials using Different Standard Test Methods. Building and Environment, 49, 86-96.

[9] Buratti, C., Belloni, E., Lascaro, E., Anna, G., \& Ricciardi, P. (2016). Sustainable Panels with Recycled Materials for Building Applications : Environmental and Acoustic Characterization. Energy Procedia, 101(September), 972-979.

[10] Kim, J., Kim, H., Lim, D., Lee, Y., \& Kim, J. H. (2016). Effects of Indoor Air Pollutants on Atopic Dermatitis. International Journal of Environmental Research and Public Health, 13(12), 1-13.

[11] Weschler, C. J. (2009). Changes in Indoor Pollutants Since the 1950s. Atmospheric Environment, 43(1), 153-169.

[12] Moffat, D. W. (1997). Handbook of Indoor Air Quality Management. United States of America: Prentice Hall.

[13] United States Environmental Protection Agency (2017). Retrieved on September 9, 2017 from https://www.epa.gov/indoor-air-quality-iaq/introduction -indoor-air-quality

[14] Khan, F. I., \& Ghoshal, A. K. (2000). Removal of Volatile Organic Compounds from Polluted Air. Loss Prevention in the Process Industries, 13, 527-545 https://doi.org/10.1016/S0950-4230(00)00007-3.

[15] Liang, W., Lv, M., \& Yang, X. (2016). The Combined Effects of Temperature and Humidity on Initial Emittable Formaldehyde Concentration of a Medium-Density Fiberboard. Building and Environment, 98, 80-88. https://doi.org/10.1016/j.buildenv.2015.12.024

[16] Roffael, E. (2006). Volatile Organic Compounds and Formaldehyde in Nature, Wood and Wood Based Panels. Holz Als Roh- Und Werkstoff, 64(2), 144-149. https://doi.org/10.1007/s00107-005-0061-0

[17] Carlson, F. E., Phillips, E. K., Tenhaeff, S. C., \& Detlefsen, W. D. (1995). A Study of Formaldehyde and Other Organic Emissions from Pressing of Laboratory Oriented Strandboard. Forest products journal, 45(3), 71.
[18] Kim, K. W., Kim, S., Kim, H. J., \& Park, J. C. (2010). Formaldehyde and TVOC Emission Behaviors According to Finishing Treatment with Surface Materials using 20L Chamber and FLEC. Journal of Hazardous Materials, 177, 90-94. https://doi.org/10.1016/j.jhazmat.2009.09.060

[19] Jun, S., \& Shuang, L. (2012). Correlation of Two Environmental Chambers for Measuring VOC Emission from Wood-based Panels. In 2012 International Conference on Biobase Material Science and Engineering (pp. 99-102). https://doi.org/10.1109/BMSE.2012.6466190

[20] Liu, Y., Shen, J., \& Zhu, X., (2008). Effect of Hot-pressing Parameters on the Emission of Volatile Organic Compounds from Particleboard. Beijing Linye Daxue Xuebao/Journal of Beijing Forestry University, 30(5), 139-142.

[21] He, Z., Zhang, Y., \& Wei, W. (2012). Formaldehyde and VOC Emissions at Different Manufacturing Stages of Wood-based Panels. Building and Environment, 47, 197-204 https://doi.org/10.1109/BMSE.2012.6466190

[22] Conner, A. H. (1996). Urea-formaldehyde Adhesive Resins: Polymeric Materials Encyclopedia. 8496-8501

[23] Dunky, M. (1998). Urea formaldehyde ( UF ) Adhesive Resins for Wood. International Journal of Adhesion \& Adhesives, 18(June 1997), 95-107. https://doi.org/10.1016/S0143-7496(97)00054-7

[24] Agency for Toxic Substances and Disease Registry (2015). Retrieved on September 9, 2017 from https://www.atsdr.cdc.gov/toxfaqs/tfacts111.pdf 